\title{
Uso de Passiflora edulis f. flavicarpa na redução do colesterol
}

\author{
Alessandra Teixeira Ramos ${ }^{1 *}$, Maria Auxiliadora L. Cunha ${ }^{1}$, Armando U. O. Sabaa-Srur ${ }^{3}$, \\ Vanúsia Cavalcanti F. Pires ${ }^{4}$, Maria Aparecida A. Cardoso ${ }^{1}$, Margareth de F. M. Diniz ${ }^{2}$, \\ Carla Campos Muniz Medeiros ${ }^{5}$
}

\author{
${ }^{1}$ Departamento de Farmácia, Universidade Estadual da Paraíba, Campus Universitário, Bodocongó, 58100-753, \\ Campina Grande, PB, Brasil, \\ ${ }^{2}$ Laboratório de Tecnologia Farmacêutica, Universidade Federal da Paraíba, Caixa Postal 5009, 58051-970, \\ João Pessoa, PB, Brasil, \\ ${ }^{3}$ Departamento de Nutrição Básica Experimental, Universidade Federal do Rio de Janeiro, Cidade \\ Universitária, 21949-900, Rio de Janeiro, RJ, Brasil, \\ ${ }^{4}$ Departamento de Química, Universidade Estadual da Paraíba, Campus Universitário, Bodocongó, \\ 58100-753, Campina Grande, PB, Brasil \\ ${ }^{5}$ Departamento de Enfermagem, Universidade Federal da Paraíba, Campus Universitário, \\ Bodocongó, 58100-753, Campina Grande, PB, Brasil
}

\begin{abstract}
RESUMO: A farinha da casca de maracujá é rica em uma fibra solúvel chamada pectina. Para avaliar o efeito dessa farinha na redução do colesterol foi realizado um estudo clínico piloto com dezenove mulheres, com idade entre 30 e 60 anos e apresentando hipercolesterolemia (colesterol $\geq 200 \mathrm{mg} / \mathrm{dL}$ ). Elas foram tratadas diariamente com $30 \mathrm{~g}$ da farinha da casca de maracujá por 60 dias. Após esse tempo foi observada uma redução estatisticamente significante $(\mathrm{p}<0,05)$ nos níveis colesterol total $(\mathrm{p}=0,00000)$ e colesterol LDL $(\mathrm{p}=0,01193)$. Os resultados sugerem que a farinha da casca de maracujá seja utilizada na alimentação humana, juntamente com os alimentos, ou como matéria prima na produção de outros produtos, com o objetivo de reduzir o colesterol.
\end{abstract}

Unitermos: Passiflora edulis, Passifloracea, pectina, colesterol.

\begin{abstract}
Use of Passiflora edulis $\boldsymbol{f}$. flavicarpa on cholesterol reduction". The flour of the passion fruit peel is rich in a soluble fiber called pectin. In order to evaluate the effect of this flour on cholesterol reduction, a pilot clinical study, was carried out with nineteen women, aged between 30 and 60 years, all of whom presented high cholesterol level (cholesterol $\geq 200 \mathrm{mg} / \mathrm{dL}$ ). The treatment consisted of daily doses of $30 \mathrm{~g}$ of the flour during 60 days. At the end of the 60 days it was observed a statistical reduction $(p<0.05)$ of the total cholesterol $(p=0,00000)$ and LDL-cholesterol $(p=0,01193)$ levels. The results suggested that the passion fruit peel flour, rich in pectin, can be used in human diet, with a large variety of foods, or as a raw material for other products intending to reduce cholesterol levels.
\end{abstract}

Keywords: Passiflora edulis, Passifloracea, pectin, cholesterol.

\section{INTRODUÇÃO}

Uma das principais causas de mortalidade no mundo são as doenças cardiovasculares. Seu crescimento em países desenvolvidos e/ ou em desenvolvimento tem sido relevante, causando entre outras conseqüências, o aumento dos gastos de saúde pública, diminuição da qualidade e do tempo de vida (Rique et al., 2002; Hermsdorff et al., 2004; Barbosa-Filho et al., 2006).

Há inúmeras evidências sobre a associação entre hipercolesterolemia, hipertrigliceridemia, lipoproteínas plasmáticas e doença arterial coronariana (Coronelli; Moura, 2003; Martinez et al., 2004; Gonçalves et al., 2006).

A ingestão de fibras dietéticas, encontradas principalmente em cereais, leguminosas e frutas, são uma alternativa para redução dos fatores de risco para as doenças cardiovasculares (Callegaro et al., 2005; Lairon et al., 2005). O consumo de fibras e os benefícios provenientes dessa ingesta têm levado ao desenvolvimento de produtos alimentares que contenham extratos de fibras vegetais (Harrington et al., 
2001; Castro et al., 2003; Hsu et al., 2006).

A fibra alimentar constitui numa associação complexa de diferentes polissacarídeos de plantas, resistentes a hidrólise (causada pelas enzimas secretadas pelo trato digestório dos seres humanos) e são classificadas em fibras solúveis (pectinas, gomas e mucilagens) e insolúveis (celulose, hemicelulose e lignina) de acordo com a solubilidade de seus componentes em água (Mattos; Martins, 2000).

A família Passifloraceae possui aproximadamente 16 gêneros e 650 espécies, sendo o gênero Passiflora considerado o mais importante, com cerca de 400 espécies. Essas plantas crescem essencialmente nas regiões tropicais, mas também estão presentes nas áreas subtropicais e temperadas do mundo. Várias espécies desse gênero, conhecidas vulgarmente como maracujá, têm amplo uso na medicina popular, sendo suas partes aéreas utilizadas tradicionalmente na Europa e na América no tratamento da ansiedade, insônia e irritabilidade (Petry et al., 2001; De Paris et al., 2002; Bello et al., 2002; Ritter et al., 2002; Pereira et al., 2004; Morais et al., 2005; Ribeiro et al., 2005; Carlini et al., 2006; Silva et al., 2006; Agra et al., 2007). Segundo Córdova et al. (2005) estudos têm evidenciado as propriedades funcionais da casca do maracujá, especialmente àquelas relacionadas ao teor e o tipo de fibra. Essas características e propriedades funcionais fazem com que a casca de maracujá não seja mais considerada um resíduo industrial, uma vez que pode ser utilizada na elaboração de novos produtos.

Diante do exposto e considerando a boa fonte de pectina existente na farinha da casca de maracujá, este estudo verificou o efeito dessa farinha na redução do colesterol sérico.

\section{MATERIAL E MÉTODOS}

O produto botânico de origem vegetal utilizado para o estudo incluiu a farinha da casca de maracujá (Passiflora edulis f. flavicarpa), fornecida pelo professor Armando Sabaa-Srur, docente e pesquisador da Universidade Federal do Rio de Janeiro (UFRJ).

Tratou-se de um estudo clínico piloto fase II, aprovado previamente pelo Comitê de Ética e Pesquisa da Universidade Estadual da Paraíba (parecer $\mathrm{n}^{\mathrm{o}}$ 0012.0.133.000-05) utilizando a metodologia preconizada na Portaria de $n^{\circ}$. 116/96 da Vigilância Sanitária e nas resoluções do CNS envolvendo Pesquisa em Seres Humanos a de nº . 196/96 e 251/97, e a Resolução $\mathrm{n}^{\circ} .18 / 99$ (ANVISA) que estabelece as diretrizes básicas para análise e comprovação de propriedades funcionais e ou de saúde alegadas aos alimentos e a Resolução $n^{\circ}$ 17/99 que estabelece Diretrizes Básicas para Avaliação de Risco e Segurança dos Alimentos.

Inicialmente, foram captados 52 pacientes, do sexo feminino, com idade entre 30 - 60 anos, atendidas em uma unidade pública de saúde da cidade da Campina
Grande-PB. A captação ocorreu através de convite voluntário e aleatório. Após explicações detalhadas sobre o estudo e concordando com o protocolo do mesmo, as voluntárias assinaram um termo de consentimento livre e esclarecido e eram submetidos aos exames clínicos, laboratoriais e a uma entrevista.

$\mathrm{Na}$ entrevista inicial foram observados dados pessoais, peso, altura, pressão arterial, uso de medicamentos, bebida alcoólica, prática de atividade física, etc.

Foram excluídas do estudo as pacientes que demonstraram alterações laboratoriais nos exames de análises clínicas, que revelassem disfunção hepática, renal, diabetes, grávidas, alcoólatras ou em uso de alguma medicação hipolipemiante, ou que estivessem realizando algum tipo de dieta alimentar e atividade física.

Esses resultados serviam de parâmetro para qualificar as pacientes para o estudo e eram considerados como dados iniciais da pesquisa.

Após as análises clínica e laboratorial 25 mulheres se qualificaram para o estudo. Considerouse hipercolesterolemia níveis de colesterol $\geq 200$ $\mathrm{mg} / \mathrm{dL}$, com base nas III Diretrizes de Dislipidemia e Aterosclerose (III Diretrizes de Dislipidemia e Aterosclerose, 2001).

O período do estudo foi de 60 dias sendo que o acompanhamento laboratorial e clínico foi realizado a cada trinta dias.

As pacientes recebiam semanalmente sete embalagens contendo $30 \mathrm{~g}$ cada de farinha da casca de maracujá, sendo orientadas a ingeri-la ao longo do dia juntamente com os alimentos (suco, frutas, leite, etc.). Durante todo o curso da pesquisa, as pacientes foram instruídas a ingerirem no mínimo 2 litros de água/ dia, facilitando assim acão da pectina, que é uma fibra solúvel em água, e a comunicarem aos pesquisadores qualquer sinal ou sintoma adverso que porventura apresentassem.

As análises laboratoriais (dosagens bioquímicas) foram realizadas no Laboratório do Hospital Universitário Lauro Wanderley/UFPB. A coleta de sangue foi realizada no período da manhã em jejum de 12 horas, em tempo basal $\left(\mathrm{T}_{0}\right), 30$ dias $\left(\mathrm{T}_{30}\right)$ e 60 dias $\left(\mathrm{T}_{60}\right)$. Foram determinados os níveis de Colesterol Total (CT), Colesterol HDL (HDL-C). Para a determinação de Colesterol LDL (LDL-C) foi utilizado a fórmula de Friedwald. As determinações de AST, ALT, Bilirrubina Total (BT), Bilirrubina Direta (BD), Bilirrubina Indireta (BI), Fosfatase Alcalina (FA) e GGT tiveram como objetivo avaliar o potencial hepatotóxico da farinha da casca de maracujá. A dosagem de Glicose excluiu portadores de Diabetes mellitus.

\section{Análise estatística}

Para análise descritiva dos dados utilizou-se o 
programa EpiInfo, versão 6.04. Foi aplicado o teste $t$ de Student pareado. Em todos os testes foram considerados o intervalo de confiança de $95 \%$ e o nível de significância foi de $5 \%(\mathrm{p}<0,05)$.

\section{RESULTADOS}

Das 25 pacientes incluídas no estudo, 19 (76\%) concluíram integralmente as oito semanas do estudo. As seis pacientes que abandonaram a pesquisa alegaram repulsa a farinha devido ao sabor residual forte e desconfortos abdominais. Na tabela 1 , estão descritas as características físicas basais das pacientes, e após as oito semanas do estudo. A média da idade e da altura foi de $45 \pm 8$ anos e 1,55 $\pm 0,064 \mathrm{~m}$, respectivamente. A análise estatística mostrou uma diminuição significante na média de peso das pacientes $(\mathrm{p}=0,0000)$. A média basal e após 60 dias do Índice de Massa Corporal (IMC) revelaram sobrepeso das pacientes segundo a Associação Brasileira para o Estudo da Obesidade e da Síndrome Metabólica (ABESO) (http://abeso.org.br). Na Tabela 2 encontram-se os resultados referentes ao CT, HDL-C e LDL-C. Ao comparar os valores médios basais do CT $(257,31 \pm 28,01)$ e LDL-C $(163,10 \pm 27,52)$, observa-se uma redução dessas variáveis, após oito semanas $(210,36$ $\pm 35,51$ e $129,47 \pm 45,00$ ), respectivamente, observa-se ainda que essa redução ocorreu já nas primeiras quatro semanas do estudo $(225,63 \pm 36,30$ e $141,10 \pm 48,54)$. Em relação ao HDL-C não houve diferença significativa $(\mathrm{p}=0,66173)$. As demais dosagens bioquímicas como $\gamma$-GGT, FA, BT, BD, BI, ALT, AST e Glicose não apresentaram diferença significativa após 60 dias da pesquisa (Tabela 3 ).

O questionário de reações adversas mostrou que a ocorrência de sonolência e náusea foram as reações mais significativas relatadas pela pacientes. A ocorrência de sonolência esteve presente em $47,3 \%$ das pacientes após 30 e 60 dias enquanto que e a ocorrência de náusea ocorreu em $42,10 \%$ das pacientes após 30 dias e 5,2\% após 60 dias.

\section{DISCUSSÃO}

Apesar da falta de comprovação científica, a farinha da casca de maracujá tem sido alvo de grandes especulações no que diz respeito ao seu uso na diminuição do colesterol plasmático. Inúmeras reportagens e entrevistas têm sido veiculadas na mídia evidenciando o seu poder no combate a gordura do sangue.

A despeito da ampla utilização da farinha da casca de maracujá, não foi relatado até o momento nenhum trabalho clínico comprovando a ação da mesma na redução do colesterol. Portanto, a escassez de ensaios clínicos nos leva a discutirmos nossos resultados baseando-se em trabalhos que utilizaram outras fontes de fibra solúvel, como a pectina.

Estudo pré-clínico utilizando farinha da casca de maracujá na alimentação de ratos normais e diabéticos evidenciou uma redução da glicemia após quatro semanas de estudo (Junqueira et al., 2002). Em um outro trabalho pré-clínico Chau e Huang (2005) observaram uma diminuição dos níveis de triglicerídios, colesterol sérico e hepático ao utilizar em Hamsters fibra da farinha das sementes de Passiflora edulis, sugerindo o uso da farinha como fonte de fibra. A ação da pectina como agente hipocolesterolêmico em animais também foi evidenciado por vários autores.(Chandalia et al., 2000; Piedade; Canatti-Brazaca, 2003; Behall et al., 2004; Chau; Huang, 2004; Artiss et al., 2006; Fernandes et al., 2006; Hsu et al., 2006).

No presente estudo piloto observou-se uma diminuição do Colesterol Total e LDL-C ao se comparar seus valores médios basais e após oito semanas de intervenção. O período desse estudo baseou-se em outros ensaios clínicos mostrando que esse tempo é suficiente para confirmar o efeito hipolipemiante (Ballesteros et al., 2001; Rodrigues et al., 2003; Praça et al., 2004; Behall et al., 2004; Mercanligil et al., 2007).

Apesar da diminuição do Colesterol Total após oito semanas do estudo observa-se que os seus níveis se mantiveram acima do recomendado pelo III Diretrizes de Dislipidemia e Aterosclerose (colesterol $\leq 200 \mathrm{~m} /$ dL). Vale lembrar que o Programa Nacional Americano de Educação em Colesterol (NCEP - National Cholestrol Education Program) estima que para cada $1 \%$ de redução na concentração de colesterol sangüíneo, o risco de doenças cardiovasculares diminuiria em $2 \%$. Ou seja, de acordo com essas diretrizes, a terapia dietética é o primeiro passo para a diminuição do colesterol sangüíneo favorecendo a uma diminuição entre 10 - 13\% do LDL - colesterol (Martins et al., 2004).

Nesse contexto, comparando-se os valores médios antes e depois do estudo, observou-se uma redução dos níveis de LDL-C em torno de $20 \%$ sem diminuição do HDL-C. Este é um dado importante uma vez que a fração HDL do colesterol é responsável pelo seu transporte reverso do colesterol, ou seja, retira o colesterol da circulação levando-o para o fígado para ser metabolizado, por isso chamado de "bom colesterol" (Oliveira et al., 2004). Com base nesses resultados podemos afirmar que a farinha da casca de maracujá diminui o colesterol LDL sem alterar a fração HDL.. A redução do colesterol sangüíneo também foi observada por Chandalia et al. (2000) que verificaram que o consumo de $50 \mathrm{~g}$ de fibra, sendo $25 \mathrm{~g}$ de fibras solúveis e $25 \mathrm{~g}$ de fibras insolúveis, diminuiu a concentração de lipídios séricos em indivíduos diabéticos tipo 2 . Resposta semelhante foi observada por Ballesteros et al. (2001), ao introduzir $48 \mathrm{~g} /$ dia e $27 \mathrm{~g} /$ dia de fibra solúvel, respectivamente, em dois grupos de 19 homens.

Aller et al. (2004) mostraram que um modesto aumento de ingestão de fibra solúvel também diminuiu os teores de LDL - C sem diminuição dos níveis de HDL-C. 
Tabela 1. Valores basais (idade, altura, peso, IMC) e diferença entre as médias do peso e altura das pacientes após 30 e 60 dias do estudo.

\begin{tabular}{llccc}
\hline & \multicolumn{2}{c}{ Avaliação } & \multicolumn{2}{c}{$\begin{array}{c}\text { Diferença entre as médias } \\
\text { Basal/30dias }\end{array}$} \\
\hline $\begin{array}{l}\text { Idade } \\
\text { (anos) }\end{array}$ & Basal & $45,47 \pm 8,09$ & & \\
$\begin{array}{c}\text { Altura } \\
\left(\mathrm{m}^{2)}\right.\end{array}$ & Basal & $1,55 \pm 0,06$ & & \\
Peso & Basal & $73,76 \pm 12,28$ & $1,68 \pm 1,376$ & $1,94 \pm 1,311$ \\
$(\mathrm{Kg})$ & Após 30 dias & $72,29 \pm 12,01$ & $\mathrm{p}=0,00005$ & $\mathrm{p}=0,0000$ \\
& Após 60 dias & $71,92 \pm 12,13$ & & \\
$\mathrm{IMC}$ & Basal & $24,83 \pm 4,39$ & $0,47 \pm 0,612$ & $0,68 \pm 0,582$ \\
$\left(\mathrm{Kg} / \mathrm{m}^{2}\right)$ & Após 30 dias & $24,07 \pm 4,27$ & $\mathrm{p}=0,33056$ & $\mathrm{p}=0,33056$ \\
& Após 60 dias & $23,94 \pm 4,28$ & & \\
\hline
\end{tabular}

$\mathrm{p}<0,05 \mathrm{n}=19$ média \pm DP

Tabela 2. Média e Diferença entre as médias dos valores basais e após 30 e 60 dias das variáveis CT, LDL-C e HDL-C.

\begin{tabular}{cllll}
\hline & \multicolumn{2}{c}{ Avaliação } & \multicolumn{2}{c}{ Diferença entre as médias } \\
& & & Basal/30dias & Basal/60dias \\
\hline Colesterol total & Basal & $257,31 \pm 28,01$ & $31,68 \pm 28,03$ & $46,94 \pm 29,49$ \\
$(<200 \mathrm{mg} / \mathrm{dL})$ & Após 30dias & $225,63 \pm 36,30$ & $\mathrm{p}=0,00011$ & $\mathrm{p}=0,00000$ \\
& Após 60 dias & $210,36 \pm 35,51$ & & \\
Colesterol LDL & Basal & 3 & $22,0 \pm 34,29$ & $33,63 \pm 33,30$ \\
$(<130 \mathrm{mg} / \mathrm{dL})$ & Basal & $163,10 \pm 27,53$ & $\mathrm{p}=0,01193$ & $\mathrm{p}=0,00034$ \\
& Após 30 dias & $141,10 \pm 48,54$ & & \\
Colesterol HDL & Após 60 dias & $129,47 \pm 45,00$ & & \\
$(>35 \mathrm{mg} / \mathrm{dl})$ & Basal & $39,05 \pm 5,60$ & $-0,52 \pm 5,69$ & $-0.263 \pm 2.57$ \\
& Após 30 dias & $39,15 \pm 5,39$ & $\mathrm{p}=0.69155$ & $\mathrm{p}=0.66173$ \\
\hline
\end{tabular}

$\mathrm{P}<0,05 \mathrm{n}=19$ média $\pm \mathrm{DP}$

Em relação às reações adversas citadas (sonolência e náusea), já é sabido que várias espécies do gênero Passiflora, tais como Passiflora incarnata, Passiflora alata e Passiflora edulis, são utilizadas no combate a insônia e ao estresse. Diante disso, sugerese que novos estudos sejam realizados, com objetivo de verificar a presença dos princípios ativos, na farinha da casca de maracujá, responsáveis pela propriedade sedativa, uma vez que substâncias já foram encontradas em outras partes da planta como folhas, flores, caule e polpa dos frutos (Zibadi; Watson, 2004; Yuldasheva et al., 2005; Santos et al., 2006).

De acordo com as pacientes a ocorrência de náusea era provocado pelo sabor residual forte da farinha. Estudos futuros precisam ser desenvolvidos com o propósito de melhorar o sabor, contribuindo dessa forma para uma melhor aceitabilidade da farinha.

A comparação dos níveis basais de $\gamma$ - GT, ALT, AST, fosfatase alcalina, bilirrubina total, bilirrubina direta e bilirrubina indireta com os níveis após oito semanas do estudo podem indicar ausência de efeito hepatotóxico. Esses achados não corroboram com os de Zibaldi e Watson, (2004) quando administraram extrato de folhas de $P$. edulis em animais e voluntários sadios.
Os autores observaram que houve aumento significante dos níveis séricos da $\gamma$ - GT. Em outros estudos clínicos, quatro dos nove voluntários submetidos ao estudo apresentaram altos níveis séricos de amilase e dois apresentaram valores alterados de bilirrubina direta, um dia após a ingestão de chá liofilizado das folhas de $P$. edulis.

\section{CONCLUSÃO}

A farinha da casca de maracujá reduziu os níveis de colesterol total e colesterol LDL, mas não alterou os valores de colesterol HDL.

Considerando as limitações desse estudo tais como tamanho da amostra, aceitabilidade da farinha e população estudada faz-se necessários estudos clínicos mais detalhados no sentido de comprovar a ação dessa farinha sobre os lipídios séricos, tornando-a uma alternativa terapêutica viável e segura no combate a hipercolesterolemia.

\section{REFERÊNCIAS}

Agra MF, França PF, Barbosa-Filho JM 2007. Synopsis of the 
Tabela 3. Média e diferença entre as médias dos valores basais e após 30 e 60 dias, de intervenção, das variáveis ( $\gamma$ - GT, ALT, AST, FA, BT, BD, BI e Glicose).

\begin{tabular}{|c|c|c|c|c|}
\hline & \multicolumn{2}{|c|}{ Avaliação } & \multicolumn{2}{|c|}{ Diferenca entre as médias } \\
\hline & & & Basal/30dias & Basal/60dias \\
\hline$\gamma-\mathrm{GT}$ & Basal & $28,57 \pm 7,58$ & $-0,158 \pm 5,03$ & $-0,105 \pm 4,02$ \\
\hline \multirow[t]{2}{*}{$(7-32 \mathrm{U} / \mathrm{L})$} & Após 30dias & $28,73 \pm 8,25$ & $\mathrm{p}=0,89281$ & $\mathrm{p}=0,91053$ \\
\hline & Após 60 dias & $28,68 \pm 7,46$ & & \\
\hline ALT & Basal & $28,68 \pm 6,16$ & $1,105 \pm 7,90$ & $0,105 \pm 6,81$ \\
\hline \multirow[t]{2}{*}{ (até $49 \mathrm{U} / \mathrm{L}$ ) } & Após 30 dias & $27,57 \pm 6,32$ & $\mathrm{p}=0,55001$ & $\mathrm{p}=0,94706$ \\
\hline & Após 60 dias & $28,57 \pm 5,17$ & & \\
\hline AST & Basal & $27,21 \pm 5,53$ & $1,789 \pm 6,37$ & $2,474 \pm 5,39$ \\
\hline \multirow[t]{2}{*}{$(7-45 \mathrm{U} / \mathrm{L})$} & Após 30 dias & $25,42 \pm 6,47$ & $p=0,23678$ & $p=0,06117$ \\
\hline & Após 60 dias & $24,7 \pm 4,71$ & & \\
\hline Fosfatase & Basal & $82,42 \pm 31,24$ & $6,474 \pm 20,08$ & $6,158 \pm 17,61$ \\
\hline Alcalina & Após 30dias & $75,94 \pm 27,83$ & $p=0,17710$ & $p=0,14485$ \\
\hline$(30-100 \mathrm{U} / \mathrm{L})$ & Após 60 dias & $76,26 \pm 24,94$ & & \\
\hline Bilirrubina total & Basal & $0,64 \pm 0,19$ & $-0,053 \pm 0,22$ & $0,053 \pm 0,22$ \\
\hline \multirow[t]{2}{*}{ (até $1,2 \mathrm{mg} / \mathrm{dL}$ ) } & Após 30 dias & $0,71 \pm 0,20$ & $p=0,33056$ & $\mathrm{p}=0,33056$ \\
\hline & Após 60 dias & $0,63 \pm 0,04$ & & \\
\hline \multirow{3}{*}{$\begin{array}{l}\text { Bilirrubina Direta } \\
\text { (até } 0,4 \mathrm{mg} / \mathrm{dL} \text { ) }\end{array}$} & Basal & $0,23 \pm 0,08$ & $-0,0047$ & $-0,0042$ \\
\hline & 30 dias & $0,27 \pm 0,13$ & & \\
\hline & 60 dias & $0,27 \pm 0,11$ & & \\
\hline \multirow{3}{*}{$\begin{array}{l}\text { Bilirrubina Indireta } \\
\text { (até } 0,8 \mathrm{mg} / \mathrm{dL} \text { ) }\end{array}$} & Basal & $0,41 \pm 0,15$ & $-0,005$ & 0,0032 \\
\hline & 30dias & $0,42 \pm 0,13$ & & \\
\hline & 60 dias & $0,38 \pm 0,15$ & & \\
\hline \multirow{3}{*}{$\begin{array}{l}\text { Glicose } \\
(70-110 \mathrm{mg} / \mathrm{dL})\end{array}$} & Basal & $87,10 \pm 10,30$ & $-1,053 \pm 8,482$ & $-1,579 \pm 8,546$ \\
\hline & Após 30 dias & $88,15 \pm 7,74$ & $\mathrm{p}=0,59517$ & $\mathrm{p}=0,43113$ \\
\hline & Após 60 dias & $88,68 \pm 7,25$ & & \\
\hline
\end{tabular}

$\mathrm{p}<0,05$ média \pm DP $\mathrm{n}=19$

plants known as medicinal and poisonous in Northeast of Brazil. Rev Bras Farmacogn 17: 114-140.

Aller R, Antonio de Luis D, Izaola O, Calle F, Olmo L. Fernandez L, Arranz T, Gonzalez Hernandez JM 2004. Effect of soluble fiber intake in lipid and glucose levels in healthy subjects: a randomized clinical trial. Diabetes Res Clin Pr 65: 7-11.

Artiss JD, Brogan K, Brucal M, Moghaddam M 2006. The effects of a new soluble dietary fiber on weight gain and selected blood paramenters in rats. Metab Clin Experiment 55: 195-202.

Ballesteros MN, Cabrera RM, Saucedo MS, Yepiz-Plascencia M, Ortega MJ, Valencia ME 2001. Dietary fiber and lifestyle influence serum lipids in free living adult men. J Am Coll Nutr 20: 649-655.

Barbosa-Filho JM, Martins VKM, Rabelo LA, Moura MD, Silva MS, Cunha EVL, Souza MFV, Almeida RN, Medeiros IA 2006. Natural products inhibitors of the angiotensin converting enzyme (ACE). A review between 1980-2000. Rev Bras Farmacogn 16: 421-446.

Behall KM, Schofield DJ, Hallfrish J 2004. Lipids significantly reduced by diets containing barley in moderately hipercholesterolemic men. J Am Coll Nutr 23:55-62.

Bello CM, Montanha JA, Schenkel EP 2002. Análise das bulas de medicamentos fitoterápicos comercializados em Porto Alegre, RS, Brasil. Rev Bras Farmacogn 12: 75-83.

Callegaro MGK, Dutra CB, Huber LS, Becker LV, Rosa CS,
Kubota EM, Hecktheur LH 2005. Determinação da fibra alimentar insolúvel,solúvel e total de produtos derivados do milho 2005. Cienc Tecnol Aliment 25: 271-274.

Carlini EA, Rodrigues E, Mendes FR, Tabach R, Gianfratti B 2006. Treatment of drug dependence with Brazilian herbal medicines. Rev Bras Farmacogn 16: 690-695.

Castro JA, Tirapegui J, Benedicto ML 2003. Effects of diet suplementation with three soluble polysaccharides on serum lipids levels of hypercholesterolemic rats. Food Chem 73: 263-269.

Chandalia M, Garg A, Lutjohann D, Bergaman KV, Grundy SM, Brinkley LJ 2000. Beneficial effects of high dietary fiber intake in patients with type 2 diabetes mellitus. New Eng J Med 342: 1392-1398.

Chau CF, Huang YL 2004. Characterization of passion fruit seeds fiber - a potential fiber source. Food Chem 85:189-194.

Chau CF, Huang YL 2005. Efects of the insoluble fiber derived from Passiflora edulis seed on plasma and hepatic lipids and fecal output. Mol Nutr Food Res 49: 786-790.

Córdova KV, Gama TMT, Winter CMG, Kaskantzis Neto G, Freitas RJS 2005. Características físico-químicas da casca de maracujá amarelo (Passiflora edulis flavicarpa Deg) obtida por secagem. Boletim CEPPA 23: 221-230.

Coronelli CLS, Moura EC 2003. Hipercolesterolemia em 
escolares e seus fatores de risco. Rev Saúde Pública 37: 24-31.

De Paris F, Petry RD, Reginatto FH, Gosmann G, Quevedo J, Salgueiro JB, Kapczinski F, Ortega GG, Schenkel EP 2002. Pharmacochemical study of aqueous extracts in Passiflora alata Dryander and Passiflora edulis Sims. Acta Farma Bonarense 21: 5-8.

III Diretrizes Brasileiras sobre Dislipidemias e Aterosclerose 2001.Publicação do Departamento de Aterosclerose da Sociedade Brasileira de Cardiologia. Data final de elaboração da versão: 23 de agosto de 2001.

Fernandes LR, Xisto MD, Penna MG, Matosinhos JM, Leal MC, Portugal LR, Leite JIA 2006. Efeito da goma guar parcialmente hidrolisada no metabolismo dos lipídios e na aterogênese de camundongos. Rev Nutr 10: 563-571.

Gonçalves MCR, Diniz MFFM, Dantas AHG, Borba JDC 2006. Solanum melongena L.) em mulheres com dislipidemias, sob controle nutricional. Rev Bras Farmacogn 16: 656-663.

Harrington ME, Flynn A, Cashman KD 2001. Effects of dietary fiber extracts on calcium absorption in the rat 2001. Food Chem 73: 263-269.

Hermsdorff HHMM, Peluzio MCG, Franceschini SCC, Priore SE 2004. Evolução histórica dos valores de referência para perfil lipídico: o que mudou e por quê. Rev Bras Nutr 9: 86-93.

Hsu PK, Chien PJ, Chen CH, Chau CF 2006. Carrot insoluble fiber-rich fraction lowers lipid and cholesterol absorption in hamsters. LWT 39: 37-342. http://www. abeso.org.br, acessada em agosto de 2007.

Junueira-Guertzenstein SM, Srur AUOS 2002. Uso da casca de maracujá (Passifloa edulis f. flavicarpa Deg) cv amarelo na alimentação de ratos (Rattus novegicus) normal e diabéticos. Revista Cadernos do Centro Universitário São Camilo 10: 213-218.

Lairon D, Arnault N, Bertrais S, Planells R, Clero E, Hercberg S, Boutron-Ruault MC 2005. Dietary fiber intake and risk factors for cardiovascular disease in french adults. Am J Clin Nutr 82: 1185-1194.

Martinez S, Zegers M, Stockins B, Bustos L, Sanhueza A, Rivera A, Soto L, Mackay A, Vega D, Rapimán P, Atton R, Alberti G 2004. Evaluación de uma maniobra nutricional tendiente a reducir los niveles de colesterol em pacientes portadores de enfermedad coronária em el sistema público de salud chileno. Rev Med Chile 132: 1457-1465.

Martins SLC, Silva HF, Novaes MRCG, Ito MR 2004. Efeitos terapêuticos dos fitosteróis e fotostanóis na colesterolemia. ALAN 54: 257-263.

Morais SM, Dantas JDP, Silva ARA, Magalhães EF 2005. Plantas medicinais usadas pelos índios Tapebas do Ceará. Rev Bras Farmacogn 15: 169-177.

Matos LL, Martins IS 2000. Consumo de fibras alimentares em população adulta. Rev Saúde Pública 34: 50-55.

Mercanligil SM, Arslan P, Alasalvar C, Okut E, Akgiil E, Pinar A, Geyik PO, Tokgozoglu L, Shahidi F 2007. Effects of hazelnut-enriched diet on plasma cholesterol and liporotein profiles in hypercholesterolemic adult men. Eur J Clin Nutr 61: 212-220.

Oliveira TT, Nagem TJ, Lopes RM, Moraes GHK, Ferreira Junior, DB, Silva RR, Maia JRS 2004. Efeito de diferentes doses de rutina sobre lipídeos no soro de coelhos machos e fêmeas. Rev Bras Anal Clin 36: 213-215.

Pereira RC, Oliveira MTR, Lemos GCS 2004. Plantas utilizadas como medicinais no município de Campos de Goytacazes - RJ. Rev Bras Farmacogn 14 (Supl. 1): 37-40.

Petry RD, Regiato F, De Paris F, Gosan G, Salgueiro JB, Quevedo J, Kapczinski F, Ostega GG, Schenkel EP 2001. Comparative pharmacological study of hidroethanol extracts of Passiflora alata and Passiflora edulis leaves. Phytother Res 15: 162-167.

Piedade J, Canniatti-Brazaca SG 2003.Comparação entre o efeito do resíduo do abacaxizeiro (caules e folhas) e da pectina cítrica de alta metoxilação do no nível de colesterol sanguineo em ratos. Cienc Tecnol Aliment 23: 149-156.

Praça JM, Thomaz A, Caramelli B 2004. Eggplant (Solanum melongea) extract does not alter serum lipid levels. Arq Bras Cardiol 82: 269-272.

Ribeiro AQ, Leite JPV, Dantas-Barros AM 2005. Perfil de utilização de fitoterápicos em farmácias comunitárias de Belo Horizonte sob a influência da legislação nacional. Rev Bras Farmacogn 15: 65-70.

Rique ABR, Soares EA, Meireles CM 2002.Nutrição e exercício na prevenção e controle das doenças cardiovasculares. Rev Bras Med Esporte 8: 244-254.

Ritter MR, Sobierajski GR, Schenkel EP, Menth LA. Plantas usadas como medicinais no município de Ipê, RS, Brasil. Rev Bras Farmacogn 12: 51-62.

Rodrigues HG, Diniz YS, Faine LA, Almeida JA, Fernandes AAH, Novelli ELB 2003. Suplementação nutricional com antioxidantes naturais: efeito da rutina na concentração de colesterol - HDL. Rev Nutr 16: 315-320.

Santos KC, Kurtz SMTF, Mullar SD, Bravatti MW, Oliveira RMMWO, Santos CAM 2006. Effects of methanolic extract from the Leaves of Passiflora actinia. Braz Arch Biol Technol 49: 565-573.

Silva MIG, Gondim APS, Nunes IFS, Sousa FCF 2006. Utilização de fitoterápicos nas unidades básicas de atenção à saúde da família no município de Maracanaú (CE). Rev Bras Farmacogn 16: 455-462.

Yudascheva LN, Carvalho EB, Catanho MTJA, Krasilnikov OV 2005. Cholesterol-depedent hemolytic activity of Passiflora quadrangularis leaves 2005. Braz J Med Biol Res 38: 1061-1070.

Zibaldi S, Watson RR 2004. Passion fruit (Passiflora edulis) composition, efficacy and safety. Evid Based Integrative Med 1: 183-187. 